\title{
Operator Equations and Inverse Problems
}

\subsection{DEFINITION OF QUASIMONOTONICITY, THE UNIQUENESS THEOREM}

The investigation of inverse problems for differential equations frequently reduces to the investigation of the first type operator equations. Such operators may come up as functionals of the solutions of some differential equation correspond to this equation. The other words, the operator is defined as the set of equations and its values are functionals of the solutions of these equations. It is often found that the equation is defined simply by one real function belonging to some class. So the operator of the inverse problem is defined at the set of the functions. The operators of inverse problems may be of complex nature; they are nonlinear as a rule. Some of them have the property that if one of the functions is larger than another in a subset of the domain of definition of these functions, then images of these functions are different.

It was this property which was used for the proof of the solution uniqueness of onedimensional inverse problems of electroprospect in the paper of (Tikhonov, 1949). In the multidimensional case (Berezanskii, 1958) applied this property for the proof of the solution uniqueness of the inverse problem of the Schrödinger equation in the class of the piecewise analytical functions. We pick out the operators by such a definition.

Let $E$ be a set of elements $x$, and let $\{\lambda\}_{E}$ be some set of real functions $\lambda(x)$ in $E$. Suppose that an element $\tau$ belonging to some set $\{\tau\}$ corresponds to every function $\lambda(x) \in\{\lambda\}_{E}$ by $\tau=\mathbf{M} \lambda$, where $\mathbf{M}$ is some operator.

As a covering of set $E$ we understand totality $\{\omega\}$ of sets $\omega \in E$, union of which is equal to $E$.

Definition. Operator $\mathbf{M}$ is called quasimonotonic with respect to the covering $\{\omega\}$ of set $E$, if it follows from inequality $\lambda_{1}(x)>\lambda_{2}(x)$ holding for all $x$ belonging to, at least, one non-empty set $\omega \in\{\omega\}$ that $\mathbf{M} \lambda_{1} \neq \mathbf{M} \lambda_{2}, \lambda_{i}(x) \in\{\lambda\}_{E}, \mathrm{i}=1,2$.

Lemma 1.1. If for every $\tau_{0} \in\{\tau\}$ the equation $\mathbf{M} \lambda=\tau_{0}$ has a unique solution, then the operator $\mathbf{M}$ is quasimonotonic with respect to any covering $\{\omega\}$ of set $E$.

This statement is obvious.

Our main purpose is to show that in special cases it follows from being quasimonotonic with respect to a fixed covering that the solution of the equation $\mathbf{M} \lambda=\tau_{0}$ is unique. 
Let $\mathbf{R}^{n+1}$ be an $(n+1)$ - dimensional real Euclidean space for $(x, y), x \in \mathbf{R}^{n},-\infty<$ $y<\infty, n \geq 0$, let $E$ and $\bar{E}$ be semispaces with $y>0$ and $y \geq 0$. Denote by $\{\lambda\}$ the set of all real infinite differentiable functions $\lambda(x, y),(x, y) \in \bar{E}$ to be quasianalytical in $y$ in this domain. Under the covering $\{\omega\}$ of the semispace $E$ we understand the set of all hemiballs $\omega(\xi, p)$ :

$$
\omega(\xi, p)=\left\{(x, y):|x-\xi|^{2}+y^{2}<p^{2}, \quad y>0\right\}, \quad \xi \in \mathbf{R}^{n}, \quad p>0 .
$$

Lemma 1.2. For every function $\lambda(x, y) \in\{\lambda\}$, which is not identically equal to zero, there exists a hemiball $\omega \in\{\omega\}$ and constants $c>0, \alpha \geq 0$ such that the inequality

$$
|\lambda(x, y)| \geq c y^{\alpha}, \quad(x, y) \in\{\omega\}
$$

holds.

Proof. Since the function $\lambda(x, y)$ is not identically equal to zero, and quasianalytical in $y$ in $\bar{E}$, the equality

$$
\left.\frac{\partial^{k} \lambda}{\partial y^{k}}\right|_{y=0}=0
$$

is impossible for all $k, k=0,1,2, \ldots$. So there exists a smallest number $m \geq 0$ and a point $\xi \in \mathbf{R}^{n}$ such that

$$
\frac{\partial^{k} \lambda(x, \mathbf{0})}{\partial y^{k}}=0, \quad k=0,1,2, \ldots, m-1, \quad x \in \mathbf{R}^{n}, \quad \frac{\partial^{m} \lambda(\xi, 0)}{\partial y^{m}} \neq 0 .
$$

If $m=0$, then the statement is obvious.

Let $m>0$. Every infinite differentiable function $\tilde{\lambda}(x, y)$ such that $\tilde{\lambda}(x, 0)=0$ (see (Malgrange, 1966)) allows a representation $\tilde{\lambda}(x, y)=y \tilde{h}(x, y)$, where

$$
\tilde{h}(x, y)=\int_{0}^{1} \frac{\partial \tilde{\lambda}(x, t y)}{\partial y} \mathrm{~d} t
$$

Combining (1.1) and (1.2) we have

$$
\lambda(x, y)=y^{m-1} h(x, y)
$$

where $h(x, y)$ is an infinite differentiable function and $h(\xi, 0) \neq 0$. Let $\bar{R}(\xi, p)$ be a closed ball in $\mathbf{R}^{n+1}$ of radius $\rho>0$, centered at $(\xi, 0)$ such that $h(x, y) \neq 0,(x, y) \in \bar{R}(\xi, p)$. By continuity $h(x, y)$ and $h(\xi, 0) \neq 0$ such ball exists.

Let $c=\min _{(x, y) \in \bar{R}(\xi, p)}|h(x, y)|$, and $\omega=R(\xi, p) \cap E$. By (1.3) we have

$$
|\lambda(x, y)|=y^{m-1}|h(x, y)| \geq c y^{m-1}, \quad(x, y) \in \omega .
$$

The Lemma is proved.

By this Lemma it follows

Theorem 1.1. Let $\mathbf{M}$ be a quasimonotonic operator with respect to the covering $\{\omega\}$ of semispace $E$ by hemiballs $\omega(\xi, p)$. If $\mathbf{M} \lambda_{1}=\mathbf{M} \lambda_{2}, \lambda_{i}(x, y) \in\{\lambda\} \subset\{\lambda\}_{E}$, then $\lambda_{1}(x, y)=\lambda_{2}(x, y)$ in $E$. 
Proof. If $\lambda_{1}(x, y) \neq \lambda_{2}(x, y)$, then by Lemma 1.2 there exists a hemiball $\omega \in\{\omega\}$ such that $\left|\lambda_{1}(x, y)-\lambda_{2}(x, y)\right| \geq c y^{\alpha}>0, \quad(x, y) \in \omega$. In particular it follows from this that in the domain $\omega$ either $\lambda_{1}>\lambda_{2}$ or $\lambda_{2}>\lambda_{1}$ holds. Since the operator $\mathbf{M}$ is quasimonotonic, $\mathbf{M} \lambda_{1} \neq \mathbf{M} \lambda_{2}$, which contradicts the condition of the theorem. The theorem is proved.

Below we will use either Theorem 1.1, or Lemma 1.2 and its variations.

\subsection{INVERSE PROBLEMS FOR HYPERBOLIC EQUATIONS}

First we will illustrate the application of Theorem 1.1 to the investigation of the uniqueness of the solution of the inverse problem for the linear hyperbolic equation, and then we proceed to more general cases.

Let $\mathbf{R}^{3}$ be an Euclidean space of $(x, y), x \in \mathbf{R}^{2},-\infty<y<\infty$. Consider the problem: in the semispace $y \geq 0$ it is required to find a strictly positive function $\lambda(x, y) \subset C^{1}\left(\mathbf{R}^{3}\right)$, even in $y$, if

1. In the domain $x \in \mathbf{R}^{2}, y \in \mathbf{R}^{1}, 0 \leq t \leq t_{0}(x, y), t_{0}>0$ there exists a unique twice differentiable solution $u(x, y, t)$ of the Cauchy problem

$$
\frac{\partial^{2} u}{\partial t^{2}}=\Delta u+\lambda(x, y) u+f(x, y, t),\left.\quad u\right|_{t=0}=\left.\frac{\partial u}{\partial t}\right|_{t=0}=0
$$

where $\Delta$ is the Laplace operator in $(x, y)$.

2. In the domain $y=0,0 \leq t \leq g_{0}(x)$ the function $\tau(x, t)=\left.u\right|_{y=0}, 0 \leq t \leq g_{0}$, $g_{0}(x)>0$ is given. The inverse problem is reduced to the investigation of the operator equation $\mathbf{M} \lambda=\tau(x, t), \lambda \in C^{1}(E), E=\{(x, y), y>0\}$. As covering $\{\omega\}$ of the semispace $y>0$ we take as above the set of all hemiballs $\omega(\xi, p): \omega(\xi, p)=$ $\left\{(x, y):|x-\xi|^{2}+y^{2}<\rho^{2}, \quad y>0\right\}, \quad \xi \in \mathbf{R}^{2}, \quad \rho>0$.

Let $\omega^{-}(\xi, p)=\left\{(x, y):|x-\xi|^{2}+y^{2}<\rho^{2}, \quad y<0\right\}$.

Theorem 1.2. If a function $f(x, y, t)$ is continuous and $f>0$, then operator $\mathbf{M}$ is quasimonotonic with respect to covering $\{\omega\}$ of domain $y>0$.

Proof. Let $\lambda_{1}(x, y)>\lambda_{2}(x, y)>0,(x, y) \in \omega_{0}=\omega\left(\xi_{0}, p_{0}\right)$. Denote by $u_{1}(x, y, t)$, $u_{2}(x, y, t)$ the solutions of the Cauchy problem corresponding to the functions $\lambda_{1}(x, y)$ and $\lambda_{2}(x, y)$. Let $\tilde{\lambda}=\lambda_{1}-\lambda_{2}, \tilde{u}=u_{1}-u_{2}$. In accordance with the Kirchhoff formula we have

$$
\tilde{u}(x, y, t)=\frac{1}{4 \pi} \int_{r \leq t} \frac{\lambda_{1}(q) \tilde{u}(q, t-r)}{r} \mathrm{~d} q+\frac{1}{4 \pi} \int_{r \leq t} \frac{\tilde{\lambda}(q) u_{2}(q, t-r)}{r} \mathrm{~d} q,
$$

where

$$
r^{2}=\sum_{i=1}^{2}\left(x_{i}-q_{i}\right)^{2}+\left(y-q_{3}\right)^{2}, \quad \mathrm{~d} q=\mathrm{d} q_{1} \mathrm{~d} q_{2} \mathrm{~d} q_{3}
$$

Note that, if

$$
u(x, y, t)=F(x, y, t)+\frac{1}{4 \pi} \int_{r \leq t} \frac{b(q) u(q, t-r)}{r} \mathrm{~d} q,
$$

and $\left.F\right|_{t>0}>0, b>0$, then (in any case for sufficiently small $t>0$ ) the inequality $u(x, y, t)>0$ holds. This follows, for example, from the proof of the existence of the solution $u(x, y, t)$ by the method of successive approximations. 
By the condition of the problem and prepositions we have $f(x, y, t)>0, \lambda_{1}-\lambda_{2}>0$, $(x, y) \in \omega_{0}, \lambda_{i}>0$. If $\lambda_{i}(x, y)=\lambda_{i}(x,-y)$ then $\lambda_{1}-\lambda_{2}>0,(x, y) \in \omega_{0}^{-}$. Let $t$ be such that the points $q$ and $(x, y)$ in (1.4) belong to $\omega^{-} \cup \omega_{0}$. From this using the note above we conclude that by the strict positivity of the second term in the right part of (1.4) for sufficiently small $t>0$ we have $\tilde{u}(x, y, t)>0,(x, y) \in \overline{\omega_{0} \cup \omega^{-}}$. If $y=0$ in the last inequality, we obtain $\tilde{u}(x, 0, t)>0$. This means operator $\mathbf{M}$ is quasimonotonic. The theorem is proved.

Let, as above, $\mathbf{R}^{3}$ be a three-dimensional Euclidean space for $(x, y), x \in \mathbf{R}^{2},-\infty<$ $y<\infty$ and $\Delta=\frac{\partial^{2}}{\partial y^{2}}+\sum_{i=1}^{2} \frac{\partial^{2}}{\partial x_{i}^{2}}$ is the Laplace operator.

Denote by $\{a\}$ a set of infinite differentiable functions $a(x, y)$ to be even and quasianalytical in $y$. Let $\lambda(x, y)$ and $\mu(x, y)$ be some functions belonging to set $\{a\}$, and $u(x, y, t)$ a solution of the Cauchy problem, $\lambda>0, \mu>0$,

$$
\begin{gathered}
\frac{1}{\lambda(x, y)} \cdot \frac{\partial^{2} u}{\partial t^{2}}=\Delta u+F(x, y, t, \mu(x, y), u)+f(x, y, t) \\
\left.u\right|_{t=0}=\left.\frac{\partial u}{\partial t}\right|_{t=0}=0
\end{gathered}
$$

where $F$ and $f$ are fixed infinite differentiable functions. It is well known that a solution $u(x, y, t)$ of such a problem for the more general case exists and is unique in some domain of $(x, y, t)$.

Here is considered the inverse problem: it is required to find one of the functions either $\lambda(x, y)$ or $\mu(x, y)$, if in the domain $|x|<r, 0 \leq t<g_{0}$ the function $\varphi(x, y)=\left.u\right|_{y=0}$ is known, where $u(x, y, t)$ is the solution of the problem (1.5). Here the inverse problem is also reduced to the investigation of the equations $\mathbf{M} \lambda=\varphi(x, y), \mathbf{M} \mu=\varphi(x, y)$.

Theorem 1.3. Let function $F(x, y, t, \mu, u)$ increase strictly monotonic in variables $\mu, u$ in the domain $\mu \geq 0, u \geq 0$ and $F(x, y, t, 0,0)=0$, and the function $f(x, y, t)>0$. Then the inverse problem has no more than one solution in the class $\{a\}$.

Proof. For the proof of the theorem it suffices to verify the operators $\mathbf{M} \lambda$ and $\mathbf{M} \mu$ being quasimonotonic. At first we note, that for every $\lambda(x, y) \in\{a\}$ and $\mu(x, y) \in\{a\}$ solution $u(x, y, t)$ of problem (1.5) for sufficiently small $t$ must satisfy the inequality $u(x, y, t)>0$, $t>0$.

Really, in accordance to Sobolev's formula (see (Sobolev, 1963)) we have

$$
u=\frac{1}{4 \pi} \int_{r \leq t}[f] \sigma \mathrm{d} v+\frac{1}{4 \pi} \int_{r \leq t}[F] \sigma \mathrm{d} v+\frac{1}{4 \pi} \int_{r \leq t}[u] \Delta \sigma \mathrm{d} v .
$$

(Here we use the meanings of (Sobolev, 1963))

Since $\sigma$ and $\Delta \sigma$ have an order of increasing no more than $1 / \tau$ and $\sigma>0$, it is clear by the formula, that the sign of $u$ is defined by the first term of the right part of this formula. In particular, if $f(x, y, t)>0$, then $u(x, y, t)>0$.

Denote as $\omega^{+}$and $\omega^{-}$open hemiballs of radius $\rho>0$ centered at point $\xi \in \mathbf{R}^{2},|\xi|<r$,

$$
\begin{aligned}
& \omega^{+}(\xi, p)=\left\{(x, y), y>0:|x-\xi|^{2}+y^{2}<\rho^{2}\right\} \\
& \omega^{-}(\xi, p)=\left\{(x, y), y<0:|x-\xi|^{2}+y^{2}<\rho^{2}\right\} .
\end{aligned}
$$


Let $\mu_{1}(x, y)$ and $\mu_{2}(x, y)$ be two solutions of the problem $(\lambda(x, y)$ is fixed $)$ and let $\mu_{1}(x, y)>$ $\mu_{2}(x, y),(x, y) \in \omega=\omega^{+} \cup \omega^{-}$. Denote by $u_{1}(x, y, t), u_{2}(x, y, t)$ solutions of the Cauchy problem

$$
\begin{gathered}
\frac{1}{\lambda} \cdot \frac{\partial^{2} u_{i}}{\partial t^{2}}=\Delta u_{i}+F\left(x, y, t, \mu_{i}, u_{i}\right)+f, \quad i=1,2, \\
\left.u_{i}\right|_{t=0}=\left.\frac{\partial u_{i}}{\partial t}\right|_{t=0}=0
\end{gathered}
$$

corresponding to the functions $\mu_{1}(x, y)$ and $\mu_{2}(x, y)$. Let $w=u_{1}-u_{2}$. Then we have

$$
\begin{gathered}
\frac{1}{\lambda} \cdot \frac{\partial^{2} w}{\partial t^{2}}=\Delta w+F\left(x, y, t, \mu_{1}, w+u_{2}\right)-F\left(x, y, t, \mu_{2}, u_{2}\right), \\
\left.w\right|_{t=0}=\left.\frac{\partial w}{\partial t}\right|_{t=0}=0 .
\end{gathered}
$$

Since $\mu_{1}(x, y)>\mu_{2}(x, y),(x, y) \in \omega$ and as above $u_{2}(x, y, t)>0$, by $F$ being monotonic the inequality $F\left(x, y, t, \mu_{1}, u_{2}\right)>F\left(x, y, t, \mu_{2}, u_{2}\right)$ holds. So by using, for example, Sobolev's formula, we obtain that $(x, y) \in \bar{\omega}, w(x, y, t)>0$ at least for small $t$. Setting $y=0$ in the last inequality we have $\varphi_{1}(x, t)-\varphi_{2}(x, t)=w(x, 0, t)>0$, this is in conflict with the problem condition.

Let now $\mu(x, y)$ be fixed and let $\lambda_{1}(x, y), \lambda_{2}(x, y)$ be two different solutions of the inverse problem. As above, let $w=u_{1}-u_{2}$ and $\lambda_{1}(x, y)>\lambda_{2}(x, y),(x, y) \in \omega$. Now we have

$$
\begin{gathered}
\frac{1}{\lambda_{1}} \cdot \frac{\partial^{2} w}{\partial t^{2}}=\Delta w+F\left(x, y, t, \mu_{1}, w+u_{2}\right)-F\left(x, y, t, \mu_{2}, u_{2}\right)+\left(\frac{1}{\lambda_{2}}-\frac{1}{\lambda_{1}}\right) \frac{\partial^{2} u_{2}}{\partial t^{2}} \\
\left.w\right|_{t=0}=\left.\frac{\partial w}{\partial t}\right|_{t=0}=0 .
\end{gathered}
$$

By the condition of the theorem $f(x, y, t)>0$, as is $\frac{\partial^{2} u_{2}}{\partial t^{2}}$. Therefore, by inequality $\lambda_{1}(x, y)>\lambda_{2}(x, y),(x, y) \in \omega$ we have

$$
\left(\frac{1}{\lambda_{2}}-\frac{1}{\lambda_{1}}\right) \frac{\partial^{2} u_{2}}{\partial t^{2}}>0, \quad(x, y) \in \omega
$$

which as above is in conflict with $w(x, 0, t)>0$. By Theorem 1.1 and the obtained results the statement of the theorem follows. For reduction to other problems see (Lavrent'ev, 1974).

\subsection{MULTIDIMENSIONAL INVERSE KINEMATIC PROBLEM OF SEISMICS}

Let $\mathbf{R}^{n+1}$ be the $(n+1)$ - dimensional Euclidean space for $(x, y), x \in \mathbf{R}^{n},-\infty<y<\infty$, $\mathbf{n}$ - radius vector of the sphere $\Omega=\{x,|x|=1\}$.

Consider the problem: in semispace $y \geq 0$ it is required to find an infinite differentiable function $\lambda(x, y) \in\{\lambda\}, 0<\lambda<\mathbf{M}<\infty$ such that:

1. The shortest line $\gamma(a, b, \mathbf{n}) \subset \bar{E}$ exists of metrics $\mathrm{d} s^{2}=\lambda^{2}(x, y)\left(\mathrm{d} x^{2}+\mathrm{d} y^{2}\right)$ joining points $a \mathbf{n}, b \mathbf{n},-\infty<a<\infty,-\infty<b<\infty, \mathbf{n} \in \Omega$, and 
2. In the domain $|a|<\infty,|b|<\infty, \mathbf{n} \in \Omega$ the function

$$
\tau(a, b, \mathbf{n})=\int_{\gamma(a, b, \mathbf{n})} \lambda(x, y) \sqrt{\mathrm{d} x^{2}+\mathrm{d} y^{2}} \equiv M \lambda
$$

is given.

The problem of determining the function $\lambda(x, y)$ by $\tau(a, b, \mathbf{n})$ is called the inverse kinematic problem of seismics, where $\lambda(x, y)$ is interpretated as the inverse of the velocity $\lambda=$ $1 / v(x, y)$ and $\tau(a, b, \mathbf{n})$ is the time of moving of the perturbation by $\gamma(a, b, \mathbf{n})$.

Let

$$
\lambda_{0}=\lambda(x, 0)=\lim _{\substack{a \rightarrow b=t \\ t \mathbf{n}=x}} \frac{\tau(a, b, \mathbf{n})}{|b-a|}
$$

In $\mathbf{R}^{n}$ we consider metric $\mathrm{d} s_{0}^{2}=\lambda_{0}^{2}(x) \mathrm{d} x^{2}=\lambda_{0}^{2}(x)|\mathrm{d} x|^{2}$. Denote by $\gamma^{0}(a, b, \mathbf{n})$ the shortest line of metric $\mathrm{d} s_{0}$ joining points $a \mathrm{n}, b \mathrm{n}$. Denote by

$$
\tau^{0}(a, b, \mathbf{n})=\int_{\gamma^{0}(a, b, \mathbf{n})} \lambda_{\mathbf{0}}|\mathrm{d} x|
$$

Theorem 1.4. If $\tau(a, b, \mathbf{n}) \neq \tau^{0}(a, b, \mathbf{n})$ for every $a, b$ and $\mathbf{n}$, then the inverse kinematic problem of seismic has no more than one quasianalitical solution in $y: \lambda(x, y) \in\{\lambda\}$.

Proof. Show that under condition of the theorem the operator

$$
\mathbf{M} \lambda=\int_{\gamma(\boldsymbol{a}, b, \mathbf{n})} \lambda(x, y) \sqrt{\mathrm{d} x^{2}+\mathrm{d} y^{2}}, \quad \lambda(x, y) \in\{\lambda\}_{\bar{E}}
$$

is quasimonotonic with respect to the covering $\{\omega\}$ of domain $E=\{(x, y): y>0\}$. In the same way as Theorem 1.1 the statement will be proved. Let $\omega_{0}=\omega\left(\xi_{0}, t\right)$ be a hemiball of radius $t>0$ centered at $\xi_{0} \in \mathbf{R}^{n}$ belonging to the semispace, and let $\lambda_{1}(x, y)>\lambda_{2}(x, y)$, $(x, y) \in \omega_{0}$. Denote by $\gamma_{i}$ the shortest line of metric $\mathrm{d} s_{i}^{2}=\lambda_{i}^{2}\left(\mathrm{~d} x^{2}+\mathrm{d} y^{2}\right)$, such that $\gamma_{i} \subset \omega_{0}$.

By the condition of the theorem the shortest line $\gamma_{i}$ cannot belong to the hyperplane $y=0$ totally, in contrast

$$
\tau_{i}=\int_{\gamma_{i}} \lambda_{i}(x, y) \sqrt{\mathrm{d} x^{2}+\mathrm{d} y^{2}}=\int_{\gamma_{i}^{0}} \lambda_{i}(x, 0)|\mathrm{d} x|=\tau_{i}^{0} .
$$

Using the inequality $\lambda_{1}(x, y)>\lambda_{2}(x, y),(x, y) \in \omega_{0}$ and the last remark, we have

$$
\tau_{1}=\int_{\gamma_{1}} \lambda_{1}(x, y) \sqrt{\mathrm{d} x^{2}+\mathrm{d} y^{2}}>\int_{\gamma_{1}} \lambda_{2}(x, y) \sqrt{\mathrm{d} x^{2}+\mathrm{d} y^{2}} \geq \int_{\gamma_{2}} \lambda_{2}(x, y) \sqrt{\mathrm{d} x^{2}+\mathrm{d} y^{2}}=\tau_{2},
$$

which means operator $\mathbf{M}$ is quasimonotonic. The theorem is proved.

More general results are included in the papers by (Anikonov, 1971; 1975).

REMARK . In the plane case, instead of the condition of Theorem 1.4 it may be taken as $\tau(a, b)<\tau\left(a, b_{1}\right)+\tau\left(b_{1}, b\right), a<b_{1}<b$. By the fulfilment of this inequality every interior point on the shortest line $\gamma(a, b)$ of metrics $\mathrm{d} s^{2}=\lambda^{2}(x, y)\left(\mathrm{d} x^{2}+\mathrm{d} y^{2}\right)$ cannot belong to the straight $y=0$.

For $n \geq 2$ variables $a, b$ can be changed in the domain $|a|<a_{0},|b|<b_{0}$, where $a_{0}>0$, $b_{0}>0$ are constant. 


\subsection{ON THE UNIQUENESS OF THE SOLUTION OF THE FREDHOLM AND VOLTERRA FIRST KIND INTEGRAL EQUATIONS}

As we noted above, inverse problems for differential equations reduce to the first kind operator equations, and often to the first kind integral equations. Let us consider multidimensional integral equations and prove the uniqueness theorems.

Let $\mathbf{R}^{n+1}$ be the Euclidean space for $(x, y), x \in \mathbf{R}^{n},-\infty<y<\infty, n \geq 0$, let $V$ be a compact metric set of $v$. Denote by $\omega(\xi, t), \xi \in \mathbf{R}^{n}, t>0,(n+1)$ - dimensional open set with properties:

1. $\omega(\xi, t)$ is contained in the semispace $y>0$ and unicity $\{\omega\}$ is the covering of this space.

2. $\omega(\xi, t)$ depends continuously of $\xi$ and $t$, and $\lim _{t \rightarrow 0} \omega(\xi, t)=(\xi, 0)$.

An example of the set $\omega(\xi, t)$ may be a hemiball of radius $t>0$ centered at point $\xi \in \mathbf{R}^{n}$

$$
\omega(\xi, t)=\left\{(x, y): \quad|x-\xi|^{2}+y^{2}<t^{2}, \quad \text { and } y>0\right\}
$$

Let $M(x, y, \xi, t, v)$ be a real continuous function on $\mathbf{R}^{n+1} \times \mathbf{R}^{n+1} \times V$ possessing properties

1. $M(x, y, \xi, t, v)>0,(x, y) \in \omega(\xi, t)$,

2. $M(x, y, \xi, t, v) \equiv 0,(x, y) \in \mathbf{R}^{n+1} \backslash \bar{\omega}(\xi, t)$,

where $\bar{\omega}(\xi, t)$ is closure of $\omega(\xi, t)$.

Let us consider this equation with respect to the function $\lambda(x, y) \in\{\lambda\}$ :

$$
\mathbf{M} \lambda \equiv \inf _{\boldsymbol{v}} \int_{\omega(\xi, t)} M(x, y, \xi, t, v) \lambda(x, y) \mathrm{d} x_{1} \ldots \mathrm{d} x_{n} \mathrm{~d} y=w(\xi, t) .
$$

Note, that if function $M$ does not depend on $v$, then the considered equation is the multidimensional analog of Volterra equation. In particular, if $n=0$ then $\mathbf{M} \lambda=\int_{0}^{t} M(y, t) \lambda(y) \mathrm{d} y$. Equation $\varphi(t)=\int_{0}^{t} M(y, t) \lambda(y) \mathrm{d} y$ is the first kind Volterra equation.

Theorem 1.5. If $\mathbf{M} \lambda_{1}=\mathbf{M} \lambda_{2}, \lambda_{i} \in\{\lambda\}, i=1,2$, then $\lambda_{1}(x, y)=\lambda_{2}(x, y), x \in \mathbf{R}^{n}$, $0 \leq y<\infty$.

Proof. It is sufficiently to state operator $\mathbf{M}$ being quasimonotonic, defined by (1.6), $\lambda \in C$.

As covering $\{\omega\}$ of the semispace $y>0$ we understand sets $\omega(\xi, t)$, satisfy conditions 1 and 2 .

Let $\lambda_{1}(x, y)>\lambda_{2}(x, y),(x, y) \in \omega_{0}=\omega\left(\xi_{0}, t_{0}\right), \lambda_{i} \in\{\lambda\}, i=1,2$. Since the kernel $M(x, y, \xi, t, v)$ is continuous and has properties 1 and 2 , functions

$$
w_{i}(\xi, t, v)=\int_{\omega(\xi, t)} M(x, y, \xi, t, v) \lambda_{i}(x, y) \mathrm{d} x \mathrm{~d} y, \quad i=1,2,
$$


are continuous in the domain $\xi \in \mathbf{R}^{n}, t>0, v \in V$. Thus as $V$ is a compact metric space there exist elements $v_{i} \in V$ so that

$$
w_{i}(\xi, t)=\inf _{v} w_{i}(\xi, t, v)=w_{i}\left(\xi, t, v_{i}\right), \quad i=1,2 .
$$

By the above we have

$$
\begin{aligned}
w_{1}\left(\xi_{0}, t_{0}\right) & =\int_{\omega_{0}} M\left(x, y, \xi_{0}, t_{0}, v_{1}\right) \lambda_{1}(x, y) \mathrm{d} x \mathrm{~d} y>\int_{\omega_{0}} M\left(x, y, \xi_{0}, t_{0}, v_{1}\right) \lambda_{2}(x, y) \mathrm{d} x \mathrm{~d} y \\
& \geq \int_{\omega_{0}} M\left(x, y, \xi_{0}, t_{0}, v_{2}\right) \lambda_{2}(x, y) \mathrm{d} x \mathrm{~d} y=w_{2}\left(\xi_{0}, t_{0}\right)
\end{aligned}
$$

which means operator $\mathbf{M}$ is quasimonotonic. The theorem is proved.

Suppose now that sets $\omega(\xi, t)$ have additional properties:

1. For every $h \geq 0$ set $\omega(\xi, t, h)=\omega(\xi, t) \cap\left\{y>h, \quad x \in \mathbf{R}^{n}\right\}, t>h$, is an $(n+1)$ dimensional open set,

2. $\omega(\xi, t, h)$ depends continuously of $\xi, t, h$ and $\lim _{t \rightarrow h} \omega(\xi, t, h)=(\xi, h)$.

Define a class $\{\lambda\}_{\infty}$ of $\lambda(x, y)$, given by the definition $\lambda(x, y) \in\{\lambda\}_{\infty}$, there exists some sequence of numbers $h_{i}, 0=h_{0} \leq h_{1} \leq \ldots \leq h_{m} \ldots$, and some sequence of functions $\lambda_{i}(x, y), h_{i} \leq y \leq h_{i+1}, x \in \mathbf{R}^{n}$, analytical in $y$, which are infinite differentiable with respect to $(x, y)$ such that $\lambda(x, y)=\lambda_{i}(x, y), x \in \mathbf{R}^{n}, h_{i} \leq y \leq h_{i+1}$. In other words, class $\{\lambda\}_{\infty}$ consists of functions that are piecewise analytical in $y$ and infinite differentiable with respect to $(x, y)$.

Theorem 1.6. If $\mathbf{M} \lambda_{1}=\mathbf{M} \lambda_{2}, \lambda_{i} \in\{\lambda\}_{\infty}, i=1,2$, then $\lambda_{1}(x, y)=\lambda_{2}(x, y), x \in \mathbf{R}^{n}$, $y \geq 0$.

Proof. Let $\lambda_{1}(x, y)$ and $\lambda_{2}(x, y) \lambda_{i} \in\{\lambda\}_{\infty}$ be two solutions of equation (1.6), such as $\mathbf{M} \lambda_{1}=\mathbf{M} \lambda_{2}$. By continuity of the kernel $M(x, y, \xi, t, v)$ and conditions 1 and 2 , functions

$$
w_{i}(\xi, t, v)=\int_{\omega(\xi, t)} M(x, y, \xi, t, v) \lambda_{i}(x, y) \mathrm{d} x \mathrm{~d} y
$$

are continuous.

Therefore $v$ is a compact metric space, from this in particular it follows the existence of $v_{i}$ so that

$$
w_{i}(\xi, t)=w_{i}\left(\xi, t, v_{i}\right)=\inf _{v} w_{i}(\xi, t, v) .
$$

Suppose that $\lambda_{1}(x, y) \neq \lambda_{2}(x, y)$. From this and by the definition of class $\{\lambda\}_{\infty}$ it follows the existence of the interval $[a, b], 0 \leq a<b<\infty$ so that the following conditions are true:

1. $\lambda_{1}(x, y)=\lambda_{2}(x, y), x \in \mathbf{R}^{n}, y<a$,

2. $\lambda_{i}(x, y)$ are analytical in $y$ when $a \leq y<b$, and $\lambda_{1}(x, y) \neq \lambda_{2}(x, y), x \in \mathbf{R}^{n}, a \leq y<b$.

Let $\omega^{1}(\xi, t)$ be the intersection of $\omega(\xi, t)$ with the semispace $y>0$. By Lemma 1.2 and proposition $\lambda_{1}(x, y) \neq \lambda_{2}(x, y)$ there exists a set $\omega_{0}^{1}=\omega^{1}\left(\xi_{0}, t_{0}\right)=\omega\left(\xi_{0}, t_{0}\right) \cap\{y>a\}$ so that 
1. Set $\omega_{0}^{1}$ belongs to the strip $a<y<b, x \in \mathbf{R}^{n}$,

2. $\left|\lambda_{1}(x, y)-\lambda_{2}(x, y)\right|>0, \quad(x, y) \in \omega_{0}^{1}$.

Let $\lambda_{1}>\lambda_{2},(x, y) \in \omega_{0}^{1}$. Taken this into consideration and by $\lambda_{1}=\lambda_{2}, x \in \mathbf{R}^{n}, y<a$ we have.

$$
w_{1}\left(\xi_{0}, t_{0}\right)=\int_{\omega\left(\xi_{0}, t_{0}\right)} M\left(x, y, \xi_{0}, t, v_{1}\right) \lambda_{1} \mathrm{~d} x \mathrm{~d} y>\int_{\omega\left(\xi_{0}, t_{0}\right)} M\left(x, y, \xi_{0}, t, v_{1}\right) \lambda_{2} \mathrm{~d} x \mathrm{~d} y \geq w_{2}\left(\xi_{0}, t_{0}\right) .
$$

Thus the existence of the point is shown $\left(\xi_{0}, t_{0}\right)$ with $w_{1}\left(\xi_{0}, t_{0}\right)>w_{2}\left(\xi_{0}, t_{0}\right)$. The last inequality is in conflict with the condition of the theorem. The theorem is proved.

\section{Example of nonuniqueness of a solution of the Volterra integral equation} with a positive kernel.

Let $\lambda(t) \not \equiv 0, t \geq 0, \lambda(0)=0$ be an infinite differentiable function with zero in every interval $(0, \alpha), \alpha>0$, as for example $\lambda(t)=\exp \left(-1 / t^{2}\right) \sin (1 / t), \lambda(0)=0$. Show that there exists a kernel $K(x, t)>0, x \neq 0, t \neq 0$ such that for every $x>0$ the equality

$$
\int_{0}^{x} K(x, t) \lambda(t) \mathrm{d} t=0
$$

holds.

Let $E^{+}(x), E^{-}(x)$ be sets such that

$$
\begin{aligned}
& E^{+}(x)=\{t: \lambda(t)>0\} \cap(0, x), \\
& E^{-}(x)=\{t: \lambda(t) \leq 0\} \cap(0, x)
\end{aligned}
$$

Set

$$
\begin{gathered}
\mu^{+}(x, t)=\left\{\begin{array}{ll}
1, & \text { if } t \in E^{+}(x), \\
0, & \text { if } t \in E^{-}(x),
\end{array} \quad 0 \leq t \leq x\right. \\
\mu^{-}(x, t)=\left\{\begin{aligned}
-1, & \text { if } t \in E^{-}(x), \\
0, & \text { if } t \notin E^{-}(x),
\end{aligned}\right.
\end{gathered}
$$

Introduce functions

$$
g^{+}(x)=\int_{E^{+}(x)} \lambda(t) \mathrm{d} t, \quad g^{-}(x)=\int_{E^{-}(x)} \lambda(t) \mathrm{d} t .
$$

Define kernel $K(x, t)$ by $K(x, t)=-\mu^{+}(x, t) g^{-}(x)-\mu^{-}(x, t) g^{+}(x)$. It is obvious, that $K(x, t)>0, x \neq 0$. We have

$$
\int_{0}^{x} K(x, t) \lambda(t) \mathrm{d} t=\int_{E^{+}(x)} K(x, t) \lambda(t) \mathrm{d} t+\int_{E^{-}(x)} K(x, t) \lambda(t) \mathrm{d} t=g^{+}(x) g^{-}(x)-g^{+}(x) g^{-}(x)=0 .
$$

Thus the kernel $K(x, t)$ is constructed such as for every $x, x \geq 0$

$$
\int_{0}^{x} K(x, t) \lambda(t) \mathrm{d} t=0
$$


Note that if $\tilde{\lambda}(t)$ is a quasianalytical function in the domain $t \geq 0$ then from the equality

$$
\int_{0}^{x} K(x, t) \tilde{\lambda}(t) \mathrm{d} t=0
$$

by Theorem 1.1 it follows that $\tilde{\lambda}(t)=0$.

\section{On the uniqueness of solution of the Fredholm first kind integral equation}

Here we consider the multidimensional first kind integral equation with singularity on the diagonal or with this properties after repeated differentiation. Let $\mathbf{R}^{n}$ be the $n$ - dimensional Euclidean space for $x=\left(x_{1}, x_{2}, \ldots, x_{n}\right)$, and let $\Omega, \omega$ be balls in $\mathbf{R}^{n}$ with:

$$
\Omega=\{x:|x|<R\}, \quad \omega=\{x:|x|<r\}, \quad R>r>0 .
$$

With $\xi$ we will denote the points of sphere annulus $C=\Omega \backslash \bar{\omega}$, here the overbar, as usual, defines the closure of the set. Consider first the integral equation

$$
\int_{\omega} M(x, \xi) \lambda(x) \mathrm{d} x=u(\xi), \quad \mathrm{d} x=\mathrm{d} x_{1} \mathrm{~d} x_{2} \ldots \mathrm{d} x_{n}
$$

where kernel $M(x, \xi)$ has properties:

1. $M(x, \xi)$ is given for all $x \in \mathbf{R}^{n}, \xi \in \mathbf{R}^{n}$, continuous for $\xi \neq x$.

2. For every $q \geq 0$ equality

$$
\lim _{|x-\xi| \rightarrow 0} M(x, \xi)(x-\xi)^{q}=\infty
$$

holds.

As example of such kernels we can take $M(x, \xi)=\exp (\varepsilon /|x-\xi|)|x-\xi|^{\delta}$, where $\varepsilon>0, \delta$ can be any number. For $\varepsilon=0$ such kernels take place in inverse problems of potential.

Let $\{\lambda\}_{a}$ be a set of functions $\lambda(x), x \in \bar{\omega}$, presented by $\lambda(x)=a(x) g(x)+b(x)$ where $a(x)$ is some analytical function in closed ball $\bar{\omega}$, and $g(x), b(x)$ are fixed measurable functions, so that function $g(x)$ satisfies the inequalities $0<M_{0} \leq g(x) \leq M_{1}$.

Note that if $g(x)=1, b(x)=0$ then set of function $\{\lambda\}_{a}$ includes the set of all analytical functions in $\bar{\omega}$.

Theorem 1.7. Equation (1.7) has no more than one solution $\lambda(x) \in\{\lambda\}_{a}$.

Proof. Suppose, that equation (1.7) has two solutions $\lambda_{1}(x)$ and $\lambda_{2}(x) \lambda_{1}(x) \neq \lambda_{2}(x)$, $\lambda(x) \in\{\lambda\}_{a}, i=1,2$. Denote as $a(x)=a_{1}(x)-a_{2}(x)$. We have $\int_{\omega} M(x, \xi) g(x) a(x) \mathrm{d} x=0$. Show, that if $a(x) \neq 0$ then there exists at least one point $\xi_{0} \in C$ so that $\int M\left(x, \xi_{0}\right) g(x) a(x) \mathrm{d} x \neq 0$. By this the statement of the theorem is be established. Let $s$ be a point on the sphere $S=\{x:|x|=r\}, B(s, t)$ is an $n$-dimensional open ball of radius $t>0$ centered at point $s \in S$. Denote by $\omega(s, t)$ and $C(s, t)$ the intersection $B(s, t) \cap \omega, B(s, t) \cap C$.

Show at first that there exists point $s_{0} \in S$ and numbers $t_{0}$ and $t_{1}, t_{0}>t_{1}$, so that the inequalities hold 
1. $|a(x)| \geq A(r-|x|)^{\alpha}, x \in \bar{\omega}\left(s_{0}, t_{0}\right)=\bar{\omega}_{0}$, where $A>0, \alpha>0$ are some constants,

2. $M(x, \xi) g(x) a(x) \geq k_{0}, x \in \bar{\omega} \backslash \bar{\omega}_{0}, \xi \in C\left(s_{0}, t_{0}\right), k_{0}$ is some constant.

Inequality 1 follows from Lemma 1.2, and estimate 2 follows from the continuity of functions $M(x, \xi)$, and $a(x)$, and $g(x)$.

Note that as $a(x)=a_{1}(x)-a_{2}(x)$ the inequality $a(x) \geq A(r-|x|)^{\alpha}, x \in \omega_{0}$ is true, in another case functions $a_{1}, a_{2}$ may be changed. Then by the condition the kernel $M(x, \xi)$ is continuous in domain $\xi \neq x$ and when $|x-\xi| \rightarrow 0, M(x, \xi) \rightarrow \infty$. Therefore for every $s \in S$ it exists intersection $\omega(s, t)$ and $C(s, t)$ so that $M(x, \xi)>0$ when $x \in \omega(s, t)$, $\xi \in C(s, t)$. By the above we include there exist intersections $\omega_{0}=\omega\left(s_{0}, t_{0}\right), C_{0}=C\left(s_{0}, t_{0}\right)$ with

1. $M(x, \xi)>0, x \in \omega_{0}, \xi \in C_{0}$,

2. $a(x) \geq A(r-|x|)^{\alpha}, x \in \omega_{0}$,

3. 3. $M(x, \xi) g(x) a(x) \geq k_{0}, \xi \in C_{0}, x \in \bar{\omega} \backslash \bar{\omega}_{0}$.

Using estimates we have

$$
\begin{aligned}
\int_{\omega} M(x, \xi) g(x) a(x) \mathrm{d} x & =\int_{\omega \backslash \omega_{0}} M(x, \xi) g(x) a(x) \mathrm{d} x+\int_{\omega_{0}} M(x, \xi) g(x) a(x) \mathrm{d} x \\
& \geq k_{0} v_{0}+M_{0} A \int_{\omega_{0}} M(x, \xi)(r-|x|)^{\alpha} \mathrm{d} x,
\end{aligned}
$$

where $v_{0}$ is volume of domain $\omega \backslash \omega_{0}$. Since $\lim _{x \rightarrow \xi} M(x, \xi)|x-\xi|^{q}=\infty, \int_{\omega_{0}} M(x, \xi)(r-$ $|x|)^{\alpha} \mathrm{d} x \rightarrow \infty$, when $\xi \rightarrow s_{0}$. Thus there exists a point $\xi_{0}$ with $\int_{\omega} M(x, \xi) g(x) a(x) \mathrm{d} x>0$. The theorem is proved.

Suppose now that kernel $M(x, \xi)$ is indefinite differentiable in the domain $\xi \neq x$. Denote as $D^{\beta}$ a differential operator with respect to $\xi=\left(\xi_{1}, \xi_{2}, \ldots, \xi_{n}\right)$ :

$$
D^{\beta}=\frac{\partial^{\beta_{1}+\beta_{2}+\ldots+\beta_{n}}}{\partial \xi_{1}^{\beta_{1}} \partial \xi_{2}^{\beta_{2}} \ldots \partial \xi_{n}^{\beta_{n}}} .
$$

Let $M_{\beta}(x, \xi)=D^{\beta} M(x, \xi)$. Let kernels $M_{\beta}(x, \xi)$ possess properties:

1. $M_{\beta}(x, \xi)$ satisfy condition 1 , above,

2. There exists a sequence of numbers $\left\{q_{m}\right\}, q_{m} \rightarrow \infty$ so that

$$
\lim _{x \rightarrow \xi} M_{\beta}(x, \xi)|x-\xi|^{q_{m}}=\infty, \quad|\beta|=m
$$

Consider the following equation with such kernel

$$
\int_{\omega} M(x, \xi) \lambda(x) \mathrm{d} x=u(\xi)
$$

Theorem 1.8. Equation (1.8) has no more than one solution $\lambda(x) \in\{\lambda\}_{a}$. 
Proof. If $\lambda_{i}=a_{i}(x) g(x)+b(x), i=1,2$ are two nonequal solutions of (1.8), then the equalities

$$
\int_{\omega} M_{\beta}(x, \xi) a(x) g(x) \mathrm{d} x=0, \quad m=0,1,2, \ldots
$$

hold, where $a(x)=a_{1}(x)-a_{2}(x), M_{\beta}(x, \xi)=D^{\beta} M(x, \xi),|\beta|=m$. As in the proof of Theorem 1.1 estimates state

1. $M_{\beta}(x, \xi)>0, x \in \omega_{0}^{m}, \xi \in C_{0}^{m}$,

2. $a(x) \geq A(r-|x|)^{\alpha}, x \in \omega_{0}^{m}$,

3. $M_{\beta}(x, \xi) g(x) a(x) \geq k_{m}, \xi \in C_{0}^{m}, x \in \bar{\omega} \backslash \bar{\omega}_{0}^{m}$.

From this and the inequality above follows

$$
\int_{\omega} M_{\beta}(x, \xi) a g \mathrm{~d} x \geq k_{m} v_{0}^{m}+A M_{0} \int_{\omega_{0}^{m}} M_{\beta}(x, \xi)(r-|x|)^{\alpha} .
$$

If the number $m$ is sufficiently large, then $\int_{\omega_{0}} M_{\beta}(x, \xi)(r-|x|)^{\alpha} \mathrm{d} x$, where $\xi \rightarrow s_{0}$ tends to infinity. Thus there exists a point $\xi_{0}$ such that $\int_{\omega} M_{\beta}\left(x, \xi_{0}\right) a(x) g(x) d x=0,|\beta|=m$, which is in conflict with (1.9). The theorem is proved.

The example of nonuniqueness of the solution.

Let the kernel $M(x, \xi)=1$, where $|\xi|>r,|x|<r / 2$ and let $\lambda(x) \neq 0$ be an infinite differentiable function in circle $\omega=\{x:|x| \leq r\}$ so that

1. $\lambda(x) \equiv 0, x \in\{x:|x|>r / 2\}$,

2. $\int_{|x|<\tau / 2} \lambda(x) \mathrm{d} x=0$.

It is obvious that $\int_{\omega} M(x, \xi) \lambda(x) \mathrm{d} x=0$.

\subsection{ON THE UNIQUENESS OF THE SOLUTION OF INTEGRAL EQUATIONS OF THE FIRST KIND WITH ENTIRE KERNEL}

In this section we consider the multidimensional integral equation of the first kind

$$
w(x)=\int_{\mathbf{R}^{n}} k(x, y) \lambda(y) \mathrm{d} y, \quad x \in D
$$

where $D$ is a domain in the real Euclidean space $\mathbf{R}^{n}, n \geq 1, k(x, y)$ is a complex-valued kernel in $\mathbf{R}^{n} \times \mathbf{R}^{n}$, represented in the form

$$
k(x, y)=\int_{B} \varphi(p, q) \mathrm{e}^{\mathrm{i}(p x+q y)} \mathrm{d} p \mathrm{~d} q .
$$


Here an integrand function $\varphi(p, q)$ is complex-valued, continuous in $\mathbf{R}^{n} \times \mathbf{R}^{n}$, finite with compact support $\bar{B}$.

It is known (Ronkin, 1974), that the kernel $k(x, y)$ is continue on an entire analytic function of exponential type. In the present section we give conditions to domain $B$ and function $\varphi(x, y)$, guaranteeing the uniqueness of solution $\lambda(y)$ of equation (1.10) in the class of continuous complex-valued finite functions.

Let $\alpha(t)>0, \alpha(0)=0$ be a continuous function in $\mathbf{R}^{1}, \varepsilon>0, \delta>0$ be fixed numbers and let space $R^{n}(p)$ be defined in the following way

$$
R^{n}(p)=\left\{q:(p, q), \quad q \in \mathbf{R}^{n}\right\} .
$$

Consider set

$$
\omega(p)=\left\{q: \sum_{i=1}^{n-1}\left(p_{i}-q_{i}\right)^{2}+q_{n}^{2}<\alpha\left(p_{n}\right), \quad q_{n}>0\right\} .
$$

The set $\omega(p)$ is an open hemiball in $R^{n}(p)$ of radius $\alpha\left(p_{n}\right)$ centered at $p_{0}=\left(p_{1}, p_{2}, \ldots, p_{n-1}, 0\right)$. Further we propose that domain $B$ and function $\varphi(p, q)=$ $\varphi_{1}(p, q)+\mathrm{i} \varphi_{2}(p, q)$ have properties:

1. For any $p, 0<p_{i}<\varepsilon, i=1,2, \ldots, n$, intersection $B(p)=B \cap R(p)$ is non-empty open set in $R^{n}(p)$ and $B(p) \subset \omega(p)$,

2. In a domain $\tilde{B}=B \cap\left\{(p, q): \sum_{i=1}^{n-1} p_{i}^{2}+q_{i}^{2}<\delta\right\}$ the following inequalities hold:

$$
\varphi_{1}(p, q) \geq 0, \quad \varphi_{2}(p, q) \geq 0, \quad \varphi_{1}(p, q)+\varphi_{2}(p, q)>0 .
$$

In the case where $n=1$ as example of a domain $B$ satisfying condition 1 may be the domain with

$$
B=\left\{\left(p_{1}, q_{1}\right): 0<q_{1}<\alpha\left(p_{1}\right), \quad 0<p_{1}<1\right\},
$$

where $\alpha(t)>0, \alpha(0)=0,0 \leq t \leq 1$ is a continuous function. An example of domain $B$, not satisfying condition 1 is the square

$$
B=\left\{\left(p_{1}, q_{1}\right): 0<p_{1}<1, \quad 0<q_{1}<1\right\} .
$$

Theorem 1.9. Let conditions 1 and 2 hold. Then equation (1.10) has no more than one continuous finite solution $\lambda(y)$.

Proof. It is clear from (1.10) and (1.11) that for any continuous finite solution $\lambda(y)$ of equation (1.10), function $w(x)$ is an entire analytic function. Therefore, if $w(x)=0$, $x \in D$, then $w(x)=0, x \in \mathbf{R}^{n}$. Let $w(x)=0, x \in \mathbf{R}^{n}$. Show, that $\lambda(y)=0, y \in \mathbf{R}^{n}$.

We have

$$
\int_{\mathbf{R}^{n}} k(x, y) \lambda(y) \mathrm{d} y=\int_{\mathbf{R}^{n}}\left[\int_{B} \varphi(p, q) \mathrm{e}^{\mathrm{i}(p x+q y)} \mathrm{d} p \mathrm{~d} q\right] \lambda(y) \mathrm{d} y=0 .
$$

Changing the order of integration we obtain the equality

$$
\int_{B}\left[\varphi(p, q) \mathrm{e}^{\mathrm{i}(p x+q y)} \int_{\mathbf{R}^{n}} \mathrm{e}^{\mathrm{i} q y} \lambda(y) \mathrm{d} y\right] \mathrm{d} p \mathrm{~d} q=0 .
$$


Let

$$
\tilde{\lambda}(q)=\int_{\mathbf{R}^{n}} \mathrm{e}^{\mathrm{i} q y} \lambda(y) \mathrm{d} y .
$$

By such designation equality (1.12) acquires the form

$$
\int_{B} \varphi(p, q) \mathrm{e}^{\mathrm{i} p x} \tilde{\lambda}(q) \mathrm{d} p \mathrm{~d} q=0 .
$$

Passing on to an iterated integral we come to the relation

$$
\int_{\mathbf{R}^{n}}\left[\int_{B(p)} \varphi(p, q) \tilde{\lambda}(q) \mathrm{d} q\right] \mathrm{e}^{\mathrm{i} p x} \mathrm{~d} p=0 .
$$

Here $B(p)$, as above, is the intersection of the domain $B$ by the plane $R^{n}(p)$ of variables $q$ of dimension $n$, passing through $p$ and orthogonal to space $\mathbf{R}^{n}$ of variables $p$. Let

$$
\tilde{\tilde{\lambda}}(p)=\int_{B(p)} \varphi(p, q) \tilde{\lambda}(q) d q .
$$

In this case we rewrite equality (1.14) as

$$
\int_{\mathbf{R}^{n}} \tilde{\tilde{\lambda}}(p) \mathrm{e}^{\mathrm{i} p x} \mathrm{~d} p=0
$$

Thus the Fourier transform of continuous function $\tilde{\tilde{\lambda}}(p)$ is equal to zero. Therefore $\tilde{\tilde{\lambda}}(p)=$ $0, p \in \mathbf{R}^{n}$ and we have

$$
\int_{B(p)} \varphi(p, q) \tilde{\lambda}(q) \mathrm{d} q=0 .
$$

Because under the hypothesis of the theorem the sought solution $\lambda(y)$ is finite continuous function, then it follows from (1.13) and by the Paley-Wiener theorem (Ronkin, 1974) that $\tilde{\lambda}(q)=\tilde{\lambda}_{1}(q)+\mathrm{i} \tilde{\lambda}_{2}(q)$ is an entire analytic function.

Therefore, real functions $\tilde{\lambda}_{i}(q), i=1,2$, are entire analytic functions too.

Determining the imaginary and real parts in (1.15) we obtain equation system

$$
\begin{aligned}
& \int_{B(p)}\left(\varphi_{1} \tilde{\lambda_{1}}-\varphi_{2} \tilde{\lambda_{2}}\right) \mathrm{d} q=0, \\
& \int_{B(p)}\left(\varphi_{1} \tilde{\lambda_{2}}+\varphi_{2} \tilde{\lambda_{1}}\right) \mathrm{d} q=0,
\end{aligned}
$$

Show that $\tilde{\lambda}_{1}(q)=0, \tilde{\lambda}_{2}(q)=0, q \in \mathbf{R}^{n}$. From here by virtue of $(1.13)$ equality $\lambda(y)=0$, $y \in \mathbf{R}^{n}$ follows. We first prove the statement concerning the analytic functions.

Let $p_{0}=\left(p_{1}^{0}, p_{2}^{0}, \ldots, p_{n-1}^{0}, 0\right)$ be a fixed point, $r>0$ be a fixed number and $\omega$ be an open hemiball of radius $r>0$ centered at $p_{0}$ :

$$
\omega_{0}=\left\{q: \sum_{i=1}^{n-1}\left(p_{i}-q_{i}^{2}\right)<r, \quad q_{n}>0\right\} .
$$

Denote as $\{\omega\}$ the set of all open hemiballs centered at $\left(p_{1}, p_{2}, \ldots, p_{n-1}, 0\right)$ and such, that $\omega<\omega_{0}$. 
If $f(q)$ is a real entire analytic function, then there exists an open hemiball $\tilde{\omega} \in\{\omega\}$ such, that for all $q \in \tilde{\omega}$ one of two inequalities holds: either $f(q)>0$, or $f(q)<0$.

Suppose, that this is not so. Then in every hemiball $\omega \in\{\omega\}$ there exists a point $q^{\omega}$ such, that $f\left(q^{\omega}\right)=0$. In particular equality follows from this

$$
\left.f(q)\right|_{q_{n}=0}=0, \quad|q|<r .
$$

Because $f\left(q^{\omega}\right)=0$ and $f\left(q_{0}^{\omega}\right)=0$, where $q_{0}^{\omega}=\left(q_{1}^{\omega}, q_{2}^{\omega}, \ldots, q_{n-1}^{\omega}, 0\right)$ then in every hemiball there exists a point $\tilde{q}^{\omega}$ such, that

$$
\left.\frac{\partial f}{\partial q_{n}}\right|_{q=\tilde{q}^{\omega}}=0, \quad \tilde{q}^{\omega} \in \omega \in\{\omega\} .
$$

This leads as above to equality $\left.\frac{\partial f}{\partial q_{n}}\right|_{q_{n}=0}=0, \quad|q|<r$.

Hence, for any $m, m \geq 0$,

$$
\left.\frac{\partial^{m} f}{\partial q_{m}^{m}}\right|_{q_{n}=0}=0, \quad|q|<r
$$

which contradicts the relation $f(q) \not \equiv 0$. The statement is proved.

If we suppose now that, although one of entire functions $\tilde{\lambda}_{1}(q), \tilde{\lambda}_{2}(q)$, satisfying equation system (1.16), is not identically equal to zero, then by virtue of the statement proved above, there exists $\tilde{\varepsilon}>0, \tilde{\varepsilon}<\varepsilon$ and a point $p$ such, that for any

$$
q \in \omega(p)=\left\{q: \sum_{i=1}^{n-1}\left(p_{i}-q_{i}\right)^{2}+q_{n}^{2}<\alpha\left(p_{n}\right), \quad q_{n}>0\right\}, \quad 0<p_{i}<\tilde{\varepsilon}, \quad i=1,2, \ldots, n,
$$

one of four relation holds

1. $\tilde{\lambda}_{1}(q) \geq 0, \tilde{\lambda}_{2}(q) \geq 0, \tilde{\lambda}_{1}(q)+\tilde{\lambda}_{2}(q)>0$,

2. $\tilde{\lambda}_{1}(q) \leq 0, \tilde{\lambda}_{2}(q) \leq 0, \tilde{\lambda}_{1}(q)+\tilde{\lambda}_{2}(q)<0$,

3. $\tilde{\lambda}_{1}(q) \leq 0, \tilde{\lambda}_{2}(q) \geq 0, \tilde{\lambda}_{1}(q)-\tilde{\lambda}_{2}(q)<0$,

4. $\tilde{\lambda}_{1}(q) \geq 0, \tilde{\lambda}_{2}(q) \leq 0, \tilde{\lambda}_{1}(q)-\tilde{\lambda}_{2}(q)>0$.

Under the hypotheses of the theorem at $\tilde{\varepsilon}<\delta$ for all $(p, q)$

$$
(p, q) \in B \cap\left\{(p, q): \sum_{1}^{n-1} p_{i}^{2}+q_{i}^{2}<\delta\right\}
$$

the following relations take place

$$
\varphi_{1}(p, q) \geq 0, \quad \varphi_{2}(p, q) \geq 0, \quad \varphi_{1}(p, q)+\varphi_{2}(p, q)>0
$$

and

$$
B(p)=B \cap R^{n}(p)<\omega(p), \quad 0<p_{i}<\tilde{\varepsilon} .
$$

That is why, together with inequalities $1-4$, at least one of equalities (1.16) cannot be performed for all $p \in \mathbf{R}^{n}$. The theorem is proved. 


\subsection{EXISTENCE AND UNIQUENESS OF A SOLUTION TO AN INVERSE PROBLEM FOR A PARABOLIC EQUATION}

Problems of finding the coefficients of differential equations from information about their solutions are called inverse problems for differential equations. As a rule, inverse problems are nonlinear. Their study, especially in the multidimensional case, is often connected with significant mathematical difficulties. We will give a method for studying solvability questions for certain nonlinear inverse problems for differential equations, using a parabolic equation as an example illustrating this method.

With the aid of the Fourier transform we are able to reduce the inverse problem under consideration to a boundary value problem for a nonlinear integro-differential equation which is fully acceptable for investigation. Methods of potential theory are applicable to the boundary value problem thus obtained; under the appropriate restrictions on the data, this leads to determination of solvability, uniqueness, and stability conditions for this inverse problem.

In a domain $Q=(-\infty, \infty) \times D, D \subset \mathbf{R}^{n}, \partial D=\Gamma_{0} \in C$, we consider the equation of parabolic type

$$
\rho(x) u_{t}-\Delta u=0 .
$$

Inverse problem. Find function $u(x, t), \rho(x)>0$ satisfying (1.17) such that

$$
\begin{gathered}
\left.u\right|_{\Gamma}=\varphi(s, t), \quad \Gamma=\Gamma_{0} \times(-\infty, \infty), \\
\left.u\right|_{t=0}=u_{0}(x), \quad x \in D,\left.\quad u_{0}(x)\right|_{x=s}=\left.\varphi\right|_{t=0}, \\
\nabla_{x} u \in L^{2}(Q), \quad u \in L^{2}\left(-\infty, \infty: W_{2}^{2}(D) \cap C(\bar{D})\right), \\
u_{t} \in L^{\infty}(-\infty, \infty): C(\bar{D}), \quad \rho(x) \in C(\bar{D}) .
\end{gathered}
$$

We introduce some notation. $G(x, y)$ is Green's function of the Dirichlet problem for the domain $D, \hat{u}(x, \xi)$ is the Fourier transform of a function $u(x, t)$ with respect to $t$ and

$$
\mu(D)=\max _{\bar{D}} \int_{D}|G(x, y)| \mathrm{d} y .
$$

First we consider problem (1.17) and (1.18).

Lemma 1.3. Let $\hat{\varphi}(\xi, s) \in W_{2}^{s / 2}\left(\Gamma_{0}\right) \cap C\left(\Gamma_{0}\right) ; \hat{\varphi} \equiv 0,|\xi|>R$, and $s \in \Gamma_{0}$. Then, for any real-valued function $\rho(x)$ such that

$$
\rho(x) \in C(\bar{D}), \quad|\rho| \leq 1 / R \mu(D)
$$

there is a unique solution to problems (1.17) and (1.18) such that $\nabla_{x} u \in L^{2}(Q), u \in$ $L^{2}\left(-\infty, \infty: W_{2}^{2}(D) \cap C(\bar{D})\right), u_{t} \in L^{\infty}((-\infty, \infty): C(\bar{D}))$ and $\hat{u}(x, \xi) \equiv 0,|\xi|>R$, $x \in \bar{D}$.

A proof of Lemma 1.3 is based on the study of the boundary value problem

$$
\Delta v+\mathrm{i} \xi \rho(x) v=0,\left.\quad v\right|_{\Gamma_{0}}=\hat{\varphi}(x, \xi), \quad x \in D,
$$

and is carried out as in (Bubnov, 1984). 
EXAMPLE. Let $A(\xi)$ and $B(\xi)$ be continuous functions, $0 \leq \xi \leq R_{0}, R_{0}>0$, let $\rho$ be a positive constant, and let $u(x, t), x, t \in \mathbf{R}^{1}$ be the function

$$
u(x, t)=\int_{0}^{R_{0}} \exp \left(x \sqrt{\frac{\xi}{2 p}}\right)\left[\cos \left(x \sqrt{\frac{\xi}{2 p}}+\xi t\right) A(\xi)+B(\xi) \sin \left(x \sqrt{\frac{\xi}{2 p}}+\xi t\right)\right] \mathrm{d} \xi
$$

that is entire analytic in $t$ and satisfies the heat equation

$$
\frac{\partial u}{\partial t}=\rho \frac{\partial^{2} u}{\partial x^{2}}
$$

According to the Paley-Wiener theorem,

$$
\hat{u}(x, \xi) \equiv 0, \quad|x|>R_{0} .
$$

Our study of the inverse problem is based on the following lemmas. Let

$$
\begin{gathered}
A_{1}=\sup _{x, \xi}\left|\int_{\Gamma_{0}} \hat{\varphi} \frac{\partial G}{\partial n} \mathrm{~d} \Gamma_{0}\right|, \\
A_{2}=\min _{\bar{D}}\left|-\int_{|\xi|<R} \mathrm{i} \xi \int \hat{\varphi} \frac{\partial G}{\partial n} \mathrm{~d} \Gamma_{0} \mathrm{~d} \xi\right|, \quad \omega_{0}=\mu(D)\left\|\Delta u_{0}\right\|_{C} R .
\end{gathered}
$$

Lemma 1.4. Let the conditions of Lemma 1.3 hold, and let

$$
\begin{aligned}
& A_{2}=\min _{\bar{D}}\left[-\int_{|\xi|<R} \mathrm{i} \xi \int_{\Gamma_{0}} \hat{\varphi} \frac{\partial G}{\partial n} \mathrm{~d} \Gamma_{0} \mathrm{~d} \xi\right]>0, \\
& A_{2} \geq \omega_{0}+R\left(8 \omega_{0} A_{1}\right)^{1 / 2}, \quad 8 R^{2} A_{1}>\omega_{0} .
\end{aligned}
$$

Then the inverse problem (1.17)-(1.20) is equivalent to the boundary value problem for the nonlinear integro-differential equation

$$
\Delta_{x} v(x, \xi)+\frac{\mathrm{i} \xi \Delta u_{0}(x, \xi)}{-\int_{|\xi|<R} \mathrm{i} \xi v(x, \xi) \mathrm{d} \xi}=0,\left.\quad v\right|_{\Gamma_{0}}=v_{0}(s, \xi)
$$

in the class of functions $v \in C(\bar{D}) \cap W_{2}^{2}(D), v(x, \xi) \equiv 0,|x|>R, x \in \bar{D}$

$$
\begin{gathered}
\min _{\bar{D}}\left[-\int_{|\xi|<R} \mathrm{i} \xi v(x, \xi) \mathrm{d} x\right] \geq R_{0}>0, \\
R_{0}=\frac{1}{3}\left[\omega_{0}+A_{2}+\left[\left(\omega_{0}-A_{2}\right)^{2}-8 R^{2} \omega_{0} A_{1}\right]^{1 / 2}\right] .
\end{gathered}
$$

Taking the nonlinearity into account and using methods of potential theory, we prove the following result. 
Lemma 1.5. Let the conditions of Lemma 1.4 be satisfied. Then there is $\delta\left(R, A_{1}, A_{2}\right)>0$ such that for any $u_{0}$ with $0<\Delta u_{0}<\delta$ there exists a unique solution $v(x, \xi)$ to problem (1.21) that belongs to the class

$$
\begin{array}{r}
B=\left\{v(x, \xi) \in C(\tilde{D}) \cap W_{2}^{2}(D), \quad v(x, \xi) \equiv 0, \quad|\xi|>R, \quad x \in \bar{D}\right. \\
\left.\sup _{x, \xi}|v(x, \xi)| \leq \frac{R_{0} A_{1}}{R_{0}-\omega_{0}}, \quad \min _{\bar{D}}\left[-\int_{|\xi|<R} \mathrm{i} \xi v(x, \xi) \mathrm{d} \xi\right] \geq R_{0}\right\} .
\end{array}
$$

From the lemmas formulated above we get our main result.

Theorem 1.10. Let the following conditions be satisfied:

$$
\begin{gathered}
\hat{\varphi}(\xi, s) \in W_{2}^{s / 2}\left(\Gamma_{0}\right) \cap C\left(\Gamma_{0}\right), \quad \hat{\varphi} \equiv 0, \quad|\xi|>R, \\
\left.\hat{\varphi}\right|_{t=0}=\left.u_{0}(x)\right|_{0}, \quad 0<\Delta u_{0} \in C(\bar{D}), \\
\min _{\bar{D}}\left[-\int_{|\xi|<R} \mathrm{i} \xi \int_{\Gamma_{0}} \hat{\varphi} \frac{\partial G}{\partial n} \mathrm{~d} \Gamma_{0} \mathrm{~d} \xi\right]=A_{2}>0, \\
A_{2} \geq \omega_{0}+R\left(8 \omega_{0} A_{1}\right)^{1 / 2}, \quad 8 R^{2} A_{1}>\omega_{0} .
\end{gathered}
$$

Then there is a $\delta\left(R, A_{1}, A_{2}\right)>0$ such that for any $u_{0}$ with $0<u_{0} \leq \delta$ the inverse problem (1.17)-(1.20) has a unique solution $(\rho, u)$; moreover,

$$
\begin{gathered}
\rho(x)=\frac{\Delta u_{0}(x, \xi)}{-\int_{|\xi|<R} \mathrm{i} v(x, \xi) \mathrm{d} \xi}, \\
u(x, t)=\int_{-\infty}^{\infty} v(x, \xi) \exp (-\xi t) \mathrm{d} x,
\end{gathered}
$$

where $v(x, \xi)$ is a solution to problem (1.21).

REMARK T. his theorem can be briefly formulated as follows: if in addition to the conditions of the first boundary value problem for a parabolic equation we require that a solution be regular in $t,-\infty<t<\infty$, then, under suitable restrictions on the data of the boundary value problem, one can also uniquely find the function $\rho(x)$ occurring in the equation, i.e. one can solve the inverse problem. From the method it is clear that this fact permits a significant generalization for other boundary value problems and other evolution equations including equation with variable coefficients. 


\subsection{ON UNIQUE SOLVABILITY OF AN INVERSE PROBLEM FOR A PARABOLIC EQUATION}

We formulate conditions for the unique solvability of the Cauchy problem for a semilinear integro-differential equation, and offer a method for its investigation (Anikonov and Belov, 1989). Such problems are reductions of inverse problems for parabolic equations in cases where the Fourier transform of their solutions with respect to chosen variables exists and some other conditions are satisfied (see (Anikonov, 1986)). Analogous inverse problems for parabolic and hyperbolic equations were investigated by different methods in (Bubnov, $1987 \mathrm{a}, \mathrm{b})$.

Let $\left(x_{1}, \ldots, x_{n-1}\right)$ be a point in the Euclidean space $\mathbf{R}^{n-1}$,

$$
\begin{aligned}
& \Pi_{\left[t_{1}, t_{2}\right]}=\left\{(t, x) \mid t_{1} \leq t \leq t_{2}, \quad x \in \mathbf{R}^{n-1}\right\} \text { a strip in } \mathbf{R}^{n}, \\
& G_{\left[t_{1}, t_{2}\right]}=\left\{(t, x, z) \mid(t, x) \in \Pi_{\left[t_{1}, t_{2}\right]}, \quad z \in \mathbf{R}^{1}\right\} \text { a strip in } \mathbf{R}^{n}, \\
& G^{\alpha}=\left\{(x, z) \mid x \in \mathbf{R}^{n-1}, \quad z \in[-\alpha, \alpha]\right\}, \\
& G_{\left[t_{1}, t_{2}\right]}^{\alpha}=\left\{(t, x, z) \mid(t, x) \in \Pi_{\left[t_{1}, t_{2}\right]}, \quad z \in[-\alpha, \alpha]\right\} \text { and } \alpha=\text { const }>0 .
\end{aligned}
$$

Let us examine the following equation in $G_{[0, T]}$ :

$$
\frac{\partial u(t, x, z)}{\partial t}=L(u(t, x, z))+\frac{\partial^{2} u(t, x, z)}{\partial t^{2}}+a(t, x) \frac{\partial u(t, x, z)}{\partial z}+q(t, x, z),
$$

where

$$
L(u)=\sum_{i, j=1}^{n-1} a_{i j} \frac{\partial^{2} u}{\partial x_{i} \partial x_{j}}+\sum_{i=1}^{n-1} b_{i} \frac{\partial u}{\partial x_{i}}+c u .
$$

We assume that $a_{i j}, b_{i}$ and $c$ are sufficiently smooth functions of $t$ given on $[0, T]$ and, in addition, $c(t) \leq 0$ and

$$
\mu|\xi|^{2} \leq \sum_{i, j=1}^{n-1} a_{i j}(t) \xi_{i} \xi_{j}, \quad \forall \xi \in \mathbf{R}^{n-1}, \quad t \in[0, T], \quad \mu=\text { const }>0 .
$$

The function $q(t, x, z)$ is given on $G_{[0, T]}$.

The function $a(t, x)(\partial a / \partial z \equiv 0)$ has yet to be found. We assume that the following condition is satisfied:

$$
\left.u\right|_{z=0}=\varphi(t, x), \quad(t, x) \in \Pi_{[0, T]},
$$

and that the Fourier transform of $u(t, x, z)$ with respect to $z$ exists:

$$
\begin{gathered}
W(t, x, y)=\frac{1}{2 \pi} \int_{-\infty}^{\infty} u(t, x, y) \mathrm{e}^{-\mathrm{i} z y} \mathrm{~d} z \\
u(t, x, y)=\int_{-\infty}^{\infty} W(t, x, y) \mathrm{e}^{\mathrm{i} z y} \mathrm{~d} y
\end{gathered}
$$

Let us introduce a Cauchy condition for equation (1.22):

$$
u(0, x, z)=u_{0}(x, z), \quad(x, z) \in \mathbf{R}^{n} .
$$


With the help of conditions (1.24) and (1.25), problem (1.22) and (1.26) can be reduced to a Cauchy problem for a integro-differential equation, not containing $a(t, x)$. Indeed, by applying the Fourier transform to equation (1.22), setting $z=0$ in (1.22) under conditions (1.24) and (1.25), we obtain the equations

$$
\begin{gathered}
\frac{\partial W}{\partial t}=L(W)-y^{2} W+\mathrm{i} y a W+Q \\
\frac{\partial \varphi}{\partial t}=L(\varphi)-\int_{-\infty}^{\infty} \lambda^{2} W \mathrm{~d} \lambda+\mathrm{i} a \int_{-\infty}^{\infty} \lambda W \mathrm{~d} \lambda+q(t, x, 0)
\end{gathered}
$$

from which we eliminate function $a$ and obtain the problem

$$
\begin{gathered}
\frac{\partial W}{\partial t}=L(W)-y^{2} W+\frac{y M}{\int_{-\infty}^{\infty} \lambda W \mathrm{~d} \lambda}\left\{\Phi+\int_{-\infty}^{\infty} \lambda^{2} W \mathrm{~d} \lambda\right\}+Q, \\
W(0, x, y)=W_{0}(x, y), \quad(x, y) \in \mathbf{R}^{n}
\end{gathered}
$$

Here

$$
Q(t, x, y)=\frac{1}{2 \pi} \int_{-\infty}^{\infty} q(t, x, y) \mathrm{e}^{-\mathrm{j} z y} \mathrm{~d} z
$$

is the Fourier transform of $q$ and

$$
\begin{gathered}
W_{0}(x, y)=\frac{1}{2 \pi} \int_{-\infty}^{\infty} u_{0}(x, y) \mathrm{e}^{-\mathrm{i} z y} \mathrm{~d} z, \\
\Phi(t, x)=\frac{\partial \varphi(t, x)}{\partial t}-L(\varphi(t, x))-q(t, x, 0) .
\end{gathered}
$$

We examine the simplest case, when $Q$ and $W_{0}$ are real-valued functions with compact supports in $y$, belonging to a finite segment $[-\alpha, \alpha]$. Then (1.27) reduces to the problem

$$
\begin{gathered}
\frac{\partial W}{\partial t}=L(W)-y^{2} W+\frac{y W}{\int_{-\alpha}^{\alpha} \lambda W \mathrm{~d} \lambda}\left\{\Phi+\int_{-\alpha}^{\alpha} \lambda^{2} W \mathrm{~d} \lambda\right\}+Q \\
W(0, x, y)=W_{0}(x, y), \quad x \in \mathbf{R}^{n-1}, \quad y \in[-\alpha, \alpha] .
\end{gathered}
$$

Equation (1.28) is a nonlinear integro-differential. It consists of partial derivatives as well as integrals of the solution with respect to the parameter, which also appears in the coefficients and the initial data of the problem. These are interesting mathematical objects which have not yet been studied. We examine the solvability of problems (1.28) and (1.29) and some properties of its solutions.

Below we denote by $C^{k, l}\left(G_{\left[t_{1}, t_{2}\right]}^{d}\right)$ the space of functions that have continuous derivatives in $G_{\left[t_{1}, t_{2}\right]}^{\alpha}$ in the space variables of order up to and including $k$, and a derivative of order $l$ in the variable $t$.

As to the input data of problem (1.28) and (1.29), we presume the following:

$$
\left\{\begin{array}{l}
\Phi, q, W_{0} \in\left(G_{[0, T]}^{\alpha}\right) ; \\
\text { all } k \text { th derivatives in } x \text { and } y \\
\text { by constants } c_{k}, \quad k=0, \ldots, 4, \text { respectively in } G_{[0, T]}^{\alpha}
\end{array}\right.
$$




$$
\begin{gathered}
Q(t, x, y) y \geq 0, \quad W_{0}(x, y) y \geq 0, \quad t \in[0 . T], \quad x \in \mathbf{R}^{n-1}, \quad y \in[-\alpha, \alpha] ; \\
I_{W_{0}}(x) \equiv \int_{-\alpha}^{\alpha} \lambda W_{0}(x, \lambda) \mathrm{d} \lambda \geq \delta>0, \quad x \in \mathbf{R}^{n-1}
\end{gathered}
$$

Let us split problem (1.28) and (1.29) according to the method of the weak approximation (Yanenko, 1971; Belov, 1986):

$$
\begin{gathered}
\frac{1}{2} \frac{\partial W_{\tau}}{\partial t}=L\left(W_{\tau}\right), \quad n \tau<t \leq\left(n+\frac{1}{2}\right) \tau \\
\frac{1}{2} \frac{\partial W_{\tau}}{\partial t}=-y^{2} W_{\tau}+\frac{y W_{\tau}}{\int_{-\alpha}^{\alpha} \lambda W_{\tau} \mathrm{d} \lambda}\left\{\Phi+\int_{-\alpha}^{\alpha} \lambda^{2} W_{\tau} \mathrm{d} \lambda\right\}+Q, \quad\left(n+\frac{1}{2}\right) \tau<t \leq(n+1) \tau, \\
W_{\tau}(0, x, y)=W_{0}(x, y), \quad(x, y) \in G^{\alpha} .
\end{gathered}
$$

Here $n=0, \ldots, N-1$ and $N_{\tau}=t_{*}, 0<t_{*} \leq T$.

We start with the Cauchy problem

$$
\begin{gathered}
\frac{1}{2} \frac{\partial v(t, x, y)}{\partial t}=\frac{y v(t, x, y)}{\int_{-\alpha}^{\alpha} \lambda v(t, x, y) \mathrm{d} \lambda}\left\{\Phi+\int_{-\alpha}^{\alpha} \lambda^{2} v(t, x, y) \mathrm{d} \lambda\right\}-y^{2} v(t, x, y)+Q(t, x, y) \\
v\left(t_{0}, x, y\right)=v_{0}(x, y), \quad(x, y) \in G^{\alpha}
\end{gathered}
$$

By (1.30'), (1.31') and (1.32') we denote conditions (1.30), (1.31), and (1.32) with $W_{0}$ replaced by $v_{0}$.

Lemma 1.6. Let $\Phi \leq k, k=$ const, and let conditions (1.30')- (1.32') be satisfied. Then there exists $\nu_{0}, 0<\nu_{0} \leq T$, such that problem (1.36) and (1.37) is uniquely solvable in the class $C^{4,1}\left(G_{\left[t_{0}, t_{0}+\nu\right]}^{\alpha}\right)$ for any $\nu \in\left(0, \nu_{0}\right]$ and any $t_{0} \in[0, T-\nu]$. $\nu_{0}$ depends only on the constants $\delta, k$ and $\alpha$.

Proof. Let us linearize problem (1.36) and (1.37) by making a shift $\theta>0$ with respect to $t$ of some terms in (1.36):

$$
\begin{aligned}
\frac{1}{2} \frac{\partial v^{\theta}(t, x, y)}{\partial t}= & \frac{y v^{\theta}(t, x, y)}{\int_{-\alpha}^{\alpha} \lambda v^{\theta}(t-\theta, x, y) \mathrm{d} \lambda}\left\{\Phi+\int_{-\alpha}^{\alpha} \lambda^{2} v^{\theta}(t-\theta, x, y) \mathrm{d} \lambda\right\} \\
- & y^{2} v^{\theta}(t, x, y)+Q(t, x, y) \\
& \left.v^{\theta}\right|_{t \leq t_{0}}=v_{0}(x, y), \quad(x, y) \in G^{\alpha} .
\end{aligned}
$$

It can be shown that there exists $\nu_{0}$ depending on $\delta, k$, and $\alpha$ such that for all $\nu \in\left(0, \nu_{0}\right]$ and $t_{0} \in[0, T-\nu]$ the solution $v^{\theta}$ of problem (1.38) exists in the interval $\left[t_{0}, t_{0}+\nu\right]$ for any fixed $\theta \in(0, \tilde{\theta}]$ and uniformly in $\theta \in(0, \tilde{\theta}]$ and $(t, x) \in \Pi_{\left[t_{0}, t_{0}+\nu\right]}$.

$$
\int_{-\alpha}^{\alpha} \lambda v^{\theta}(t-\theta, x, y) \mathrm{d} \lambda \geq \mu>0 .
$$

Estimate (1.39) allows us to obtain uniform boundedness (in $\theta$ ) of the family of solutions $\left\{v^{\theta}\right\}$ and their first derivatives with respect to $x$ and $y$. Hence, as $\theta \rightarrow 0$, uniformly 
in every compactum of $G_{\left[t_{0}, t_{0}+\nu\right]}^{\alpha}$, the functions $v^{\theta}$ converge to $v$ being solution of the problem (1.36) and (1.37) in $C^{4,1}\left(G_{\left[t_{0}, t_{0}+\nu\right]}^{\alpha}\right)$. As a result

$$
\int_{-\alpha}^{\alpha} \lambda v(t-\theta, x, y) \mathrm{d} \lambda \geq \mu>0, \quad(t, x) \in \Pi_{\left[t_{0}, t_{0}+\nu\right]} .
$$

This allows one to prove the uniqueness of the solution in the class specified above. The lemma is proved.

REMARK 1.1. In consequence of condition ( $\left.1.31^{\prime}\right)$, the inequality $v(t, x, y) y \geq 0,(t, x, y) \in$ $G_{\left[t_{0}, t_{0}+\nu\right]}^{\alpha}$ is realized.

REMARK 1.2. Let $v$ be the solution of problems (1.36), (1.37) in the interval $\left[t_{0}, t_{0}+\nu\right]$, the existence of which is guaranteed by Lemma 1.6. As follows from remark 1.1, we can apply the generalized mean value theorem (Fikhtengol'tz, 1966) to the integrals $\int_{-\alpha}^{\alpha} \lambda^{2} v \mathrm{~d} \lambda$ and $\int_{-\alpha}^{\alpha} \lambda^{3} v \mathrm{~d} \lambda$ :

$$
\int_{-\alpha}^{\alpha} \lambda^{k+1} v(t, x, \lambda) \mathrm{d} \lambda=m_{k}(t, x) \int_{-\alpha}^{\alpha} \lambda v(t, x, \lambda) \mathrm{d} \lambda, \quad k=1,2 .
$$

It is clear that $m_{k}$ are smooth functions that satisfy $-\alpha<m_{1}(t, x)<\alpha$ and $0<m_{2}(t, x)<$ $\alpha^{2}$. By multiplying (1.36) by $y$, integrating the result over $[-\alpha, \alpha]$ and then applying (1.40), we find that

$$
I_{V}(t, x) \equiv \int_{-\alpha}^{\alpha} \lambda v(t, x, \lambda) \mathrm{d} \lambda
$$

is a solution of an ordinary differential equation

$$
\frac{\mathrm{d} I_{v}(t, x)}{\mathrm{d} t}=m_{1}(t, x) \Phi(t, x)+\left(m_{1}^{2}(t, x)-m_{2}(t, x)\right) I_{v}(t, x)+I_{Q},
$$

where $x$ is a parameter. Let us consider the equation with constant coefficients

$$
\frac{\mathrm{d} j(t)}{\mathrm{d} t}=-k \alpha-\alpha^{2} j(t) .
$$

Its solution $j(t)$, satisfying the initial condition $j(0)=\delta$, is monotonic decreasing and strictly positive in an interval $\left[0, t_{*}\right]$. The value $t_{*}$ depends on $\delta, k$ and $\alpha$. Since $m_{1} \Phi \geq$ $-\alpha k$ and $m_{1}^{2}-m_{2}>-\alpha^{2}$ we have

$$
I_{V}(t, x) \geq j(t) \geq j\left(t_{*}\right), \quad t_{0} \leq t \leq t_{*},
$$

provided that

$$
I_{v}\left(t_{0}, x\right) \equiv \int_{-\alpha}^{\alpha} \lambda v_{0}(x, \lambda) \mathrm{d} \lambda \geq j\left(t_{0}\right)
$$

Theorem 1.11. Let $|\Phi| \leq k=$ const, and let conditions (1.30)-(1.32) be satisfied. Then there exists $t_{*} \in(0, T]$ such that problem (1.28) and (1.29) are uniquely solvable in $C^{2,1}\left(G_{\left[0, t_{*}\right]}^{\alpha}\right)$. The value of $t_{*}$ depends on the constants $\delta, k$, and $\alpha$. 
Proof. By applying the maximum principle to equation (1.33) (see conditions (1.22), Lemma 1.6, and the inequalities (1.42) of remark 1.2), we can easily show that a solution $W_{\tau}(t, x, y)$ of problems $(1.33)-(1.35)$ exists in the interval $\left[0, t_{*}\right]$ for any fixed $\tau \in\left(0, \tau_{0}\right]$ and, uniformly in $\tau \in\left(0, \tau_{0}\right)$,

$$
I_{W_{r}}(t, x) \geq j(t) \geq j\left(t_{*}\right), \quad t_{0} \leq t \leq t_{*}, \quad(t, x) \in \Pi_{\left[0, t_{*}\right]},
$$

where $j(t)$ is a solution of (1.41) and $j(0)=\delta$. Estimate (1.43), the maximum principle for equation (1.33), and condition (1.30) allow us to prove the estimates, uniform in $\tau \in\left(0, \tau_{0}\right]$

$$
\sum_{|k| \leq 4} \sup _{G_{\left[0, t_{*}\right]}^{\alpha}}\left|\mathbf{D}^{k} W_{\tau}\right|+\sum_{|k| \leq 2} \sup _{G_{[0, t *]}^{\alpha}}\left|\mathbf{D}_{t} \mathbf{D}^{k} W_{\tau}\right| \leq c,
$$

where $\mathbf{D}_{t}=\partial / \partial t, \mathbf{D}^{k}=\partial^{|k|} / \partial x_{1}^{k_{1}} \ldots x_{n}^{k_{n-1}} \partial y^{k_{n}}, k=\left(k_{1}, \ldots, k_{n}\right)$ is a multi-index, and $|k|=k_{1}+\ldots+k_{n}$. Because of (1.44) as in (Yanenko, 1971) and (Belov, 1986), we can prove that, uniformly for each compactum of $G_{\left[0, t_{*}\right]}^{\alpha}, W_{\tau}$ converges to $W \in C^{2,1}\left(G_{\left[0, t_{*}\right.}^{\alpha}\right)$ along with its derivatives of first and second order with respect to the space variables $x, y$, and is a solution of problem (1.28) and (1.29). The uniqueness of the solution is proved in a standard way by proving that the difference of two possible solutions of problem (1.28) and (1.29) from the class $C^{2,1}\left(G_{\left[0, t_{*}\right]}^{\alpha}\right)$ is identically zero. The theorem is proved.

REMARK 1.3. If $\Phi=0$, then we can take $k=0$, and in this case the solution $j(t)$ of equation (1.41) with the initial condition $j(0)=\delta$ is strictly positive in the whole interval $[0, T]$. This guarantees the solvability of problem (1.28) and (1.29) in this interval.

\subsection{FORMULAS IN MULTIDIMENSIONAL INVERSE PROBLEMS FOR EVOLUTION EQUATIONS}

Let $D$ be a domain in the real Euclidean space $\mathbf{R}^{n+m+1}$ of variables $(x, z, t)$, $x=\left(x_{1}, \ldots, x_{m}\right), z=\left(z_{1}, \ldots, z_{n}\right), t \in \mathbf{R}^{1}$. We consider the inverse problem of the simultaneous determination of two complex-valued functions $u(x, z, t)$ and $\lambda(x, t)\left(\partial \lambda / \partial z_{k}=0\right.$, $i=1, \ldots, n)$ in $D$ which satisfy

$$
\begin{gathered}
\frac{\partial u}{\partial t}=\mathbf{A} u+\mathbf{L} u+\lambda(x, t) \mathbf{B} u . \\
\left.u\right|_{t=0}=u_{0}(x, z),\left.\quad u\right|_{z=0}=f(x, t) .
\end{gathered}
$$

Here $\mathbf{A}, \mathbf{B}$, and $\mathbf{L}$ are linear differential operators such that $\mathbf{A}$ and $\mathbf{B}$ act with respect to $x$ and are independent of $(z, t)$ while $\mathbf{L}$ acts with respect to $z$ and is independent of $(x, t)$, the functions $u_{0}(x, z)$ and $f(x, t)$ are given, and $(x, z, t) \in D$.

As examples of operators $\mathbf{A}, \mathbf{B}$, and $\mathbf{L}$ we may consider linear differential operator with constant coefficients $\mathbf{A}_{\alpha}, \mathbf{B}_{\alpha}$, and $\mathbf{L}_{\alpha}$ such that

$$
\mathbf{A}=\sum_{\alpha} \mathbf{A}_{\alpha} \mathbf{D}_{x}^{\alpha}, \quad \mathbf{B}=\sum_{\alpha} \mathbf{B}_{\alpha} \mathbf{D}_{x}^{\alpha}, \quad \mathbf{L}=\sum_{\alpha} \mathbf{L}_{\alpha} \mathbf{D}_{z}^{\alpha} .
$$

In particular, with

$$
\mathbf{A}=\sum_{j=1}^{m} \frac{\partial^{2}}{\partial x_{j}^{2}}, \quad \mathbf{L}=\frac{\partial^{2}}{\partial z_{1}^{2}}, \quad n=1
$$


arises the inverse problem for parabolic equation, namely, to find functions $u(x, z, t)$ and $\lambda(x, t)$ satisfying

$$
\frac{\partial u}{\partial t}=\Delta u+\lambda(x, t) \mathbf{B} u,\left.\quad u\right|_{t=0}=u_{0}(x, z),\left.\quad u\right|_{z=0}=f(x, t) .
$$

For $\mathbf{B} u=\varphi(z, t)$, where $\varphi(z, t)$ is some function, the inverse problem (1.45) and (1.46) consists in finding a source function $J$ of the form $J=\lambda(x, t) \varphi(z, t)$.

We study the case when the initial state $\left.u\right|_{t=0}=u_{0}(x, z)$ can be factored in the form of a product $a(x) b(z)$, and establish explicit formulas for the solutions $u(x, z, t), \lambda(x, t)$ of (1.45) and (1.46), which introduces a constructive element in the theory of such problems. In what follows we assume that $\mathbf{A}, \mathbf{B}$, and $\mathbf{L}$ act in a well-defined way on the functions under consideration, and the integral and other transformations used are also well defined.

Lemma 1.7. Suppose that the function $f(x, t)$ such that $\mathbf{B} f \not \equiv \mathbf{0}$, that $\varphi(z, t)$ is a solution of the equation

$$
\frac{\partial \varphi}{\partial t}=\mathbf{L} \varphi
$$

and that $\varphi_{0}(t)=\varphi(0, t),(x, z, t) \in D$. Then the functions

$$
u(x, z, t)=\frac{f(x, t) \varphi(z, t)}{\varphi_{0}(t)}, \quad \lambda(x, t)=\frac{\left(\frac{\partial f}{\partial t}-\mathbf{A} f\right) \varphi_{0}-f \frac{\mathrm{d} \varphi_{0}}{\mathrm{~d} t}}{B f \varphi_{0}}
$$

satisfy equation (1.45) in $D$ and the conditions

$$
\left.u\right|_{z=0}=f(x, t),\left.\quad u\right|_{t=0}=a(x) b(z),
$$

where $a(x)=f(x, 0)$ and $b(z)=\varphi(z, 0) / \varphi_{0}(0)$.

Let $\mathbf{L}$ be a differential operator with constant coefficients and $c(\omega)$ its symbol; that is $\mathbf{L}\left(\mathrm{e}^{\mathrm{i} \omega z}\right)=c(\omega) \mathrm{e}^{\mathrm{i} \omega z}, \omega \in \mathbf{R}^{n}$.

Lemma 1.8. Let $f(x, t)$ and $q(\omega),(x, t) \in D, \omega \in \mathbf{R}^{n}$ be complex- valued functions such that the expressions

$$
\frac{\partial f}{\partial t}=\mathbf{A} f, \quad \mathbf{B} f, \quad \int_{\mathbf{R}^{n}} \mathrm{e}^{c(\omega) t+\mathrm{i} \omega z} q(\omega) \mathrm{d} \omega
$$

are meaningful and $\mathbf{B} f \not \equiv 0, \int_{\mathbf{R}^{n}} \mathrm{e}^{c(\omega) t} q(\omega) \mathrm{d} \omega \neq 0$. Then the functions

$$
\begin{gathered}
u(x, z, t)=\frac{f(x, t) \int_{\mathbf{R}^{n}} \mathrm{e}^{c(\omega) t+\mathrm{i} \omega z} q(\omega) d \omega}{\int_{\mathbf{R}^{n}} e^{c(\omega) t} q(\omega) \mathrm{d} \omega}, \\
\mathbf{B} f \int_{\mathbf{R}^{n}} \mathrm{e}^{c(\omega) t} q(\omega) \mathrm{d} \omega
\end{gathered}
$$


satisfy equation (1.45) and the conditions $\left.u\right|_{z=0}=f(x, t)$ and $\left.u\right|_{t=0}=a(x) b(z)$, where

$$
a(x)=f(x, 0), \quad b(z)=\frac{\int_{\mathbf{R}^{n}} q(\omega) \mathrm{e}^{\mathrm{i} \omega z} \mathrm{~d} \omega}{\int_{\mathbf{R}^{n}} q(\omega) \mathrm{d} \omega} .
$$

The following assertion holds.

Theorem 1.12. Suppose that $\mathbf{L}=\sum_{1}^{n} \frac{\partial^{2}}{\partial z_{k}^{2}}, z \in \mathbf{R}^{n}$, and $u_{0}(x, z)=a(x) b(z)$, in problem (1.45) and (1.46), where $a(x)=b(x, 0)$ and $b(z)$ is a continuous bounded complex-valued function in $\mathbf{R}^{n}$ with $b(0)=1$. If the conditions in Lemma 1.7 hold, then the solution $u(x, z, t), \lambda(x, t)$ of the inverse problem (1.45) and (1.46) can be represented in the form

$$
\begin{gathered}
u(x, z, t)=\frac{f(x, t) \int_{\mathbf{R}^{n}} b(y) \exp \left(-|y-z|^{2} / 4 t\right) \mathrm{d} y}{\int_{\mathbf{R}^{n}} b(y) \exp \left(-y^{2} / 4 t\right) \mathrm{d} y}, \\
\lambda(x, t)=\left\{\left(\frac{\partial f}{\partial t}-\mathbf{A} f\right) \frac{1}{(2 \pi t)^{n / 2}} \int_{\mathbf{R}^{n}} b(y) \exp \left(-\frac{y^{2}}{4 t}\right) \mathrm{d} y\right. \\
\left.-f \frac{\partial}{\partial t} \frac{1}{(2 \pi t)^{n / 2}} \int_{\mathbf{R}^{n}} b(y) \exp \left(-\frac{y^{2}}{4 t}\right) \mathrm{d} y\right\}^{-1} \\
\times\left(\mathbf{B} f \frac{1}{(2 \pi t)^{n / 2}} \int_{\mathbf{R}^{n}} b(y) \exp \left(-\frac{y^{2}}{4 t}\right) \mathrm{d} y\right)^{-} .
\end{gathered}
$$

Corollary. Suppose that $\mathbf{B} u=\varphi(z, t)$ in (1.45) and that the conditions of the Theorem 1.12 hold. Then the source function $J(x, z, t)=\varphi(z, t) \lambda(x, t)$ and the solution $u(x, z, t)$ of (1.45) can be found from the data (1.46) by means of the formulas

$$
\begin{gathered}
\varphi(z, t)=\frac{1}{(2 \pi t)^{n / 2}} \int_{\mathbf{R}^{n}} b(y) \exp \left(-|y-z|^{2} / 4 t\right) \mathrm{d} y, \\
u(x, z, t)=\frac{f(x, t) \varphi(z, t)}{\varphi_{0}(t)}, \quad \varphi_{0}(t)=\varphi(0, t), \\
J(x, z, t)=\frac{\left(\frac{\partial f}{\partial t}-\mathbf{A} f\right) \varphi_{0}-f \frac{\mathrm{d} \varphi_{0}}{\mathrm{~d} t}}{\varphi_{0}^{2}(t)} \varphi(z, t) .
\end{gathered}
$$

From Lemma 1.8 we deduce the following assertion.

Theorem 1.13. Suppose that $u_{0}(x, z)=a(x) b(z)$ in (1.45) and (1.46), where $z \in \mathbf{R}^{n}$, $a(x)=f(x, 0), b(z)$ has a Fourie transform and $b(0)=1$, and that the conditions in 
Lemma 1.8 hold. Then the solution $u(x, z, t), \lambda(x, t)$ of the inverse problem (1.45) and (1.46) is given by

$$
\begin{gathered}
u(x, z, t)=\frac{f(x, t) \int_{\mathbf{R}^{n}} \mathrm{e}^{c(\omega) t+\mathrm{i} \omega z} q(\omega) \mathrm{d} \omega}{\int_{\mathbf{R}^{n}} \mathrm{e}^{c(\omega) t} q(\omega) \mathrm{d} \omega}, \\
\lambda(x, t)=\frac{\left(\frac{\partial f}{\partial t}-\mathbf{A} f\right) \int \mathrm{e}_{\mathbf{R}^{n}} \mathrm{e}^{c(\omega) t} q(\omega) \mathrm{d} \omega-f \int_{\mathbf{R}^{n}} \mathrm{e}^{c(\omega) t} q(\omega) c(\omega) \mathrm{d} \omega}{\mathbf{B} f \int_{\mathbf{R}^{n}} \mathrm{e}^{c(\omega) t} q(\omega) \mathrm{d} \omega},
\end{gathered}
$$

where

$$
q(\omega)=\int_{\mathbf{R}^{n}} b(y) \mathrm{e}^{-\mathrm{i} z \omega} \mathrm{d} z
$$

